

\section{Las tablets como herramientas educativas para la infan- cia. Neox Kidz, un estudio de caso}

Tablets as educational tools for childhood. Neox Kidz, a case study

DOI: 10.15213/REDES.N12.P46

INMACULADA SÁNCHEZ-LABELLA MARTÍN

\section{RESUMEN}

Con el surgimiento y el desarrollo de Internet la tecnología se ha convertido en la protagonista de las sociedades desarrolladas presentando un panorama el que se cruza el plano de lo educativo y lo tecnológico. En el escenario de las nuevas pantallas las tablets se han implantado con fuerza en el día a día de los más pequeños como una herramienta de fácil manejo pero, ¿son las tablets herramientas educativas? Actualmente casi el ochenta por ciento de los niños y niñas utilizan frecuentemente este nuevo dispositivo para entretenerse el cual les concede autonomía y libertad para acceder a multitud de contenidos; preocupa, por tanto, que los más pequeños no encuentren en ellas contenidos educativos.

En este trabajo se analiza la aplicación Neox Kidz por considerarse un espacio con material audiovisual creado para la infancia. El análisis de contenido de cada uno de sus elementos permitirá demostrar que dicha app es un entorno seguro presentando los dibujos animados, las series y los programas con una finalidad educativa basada en el entretenimiento de calidad.

PALABRAS-CLAVE: TECNOLOGÍA, EDUCACIÓN, TABLETS, CALIDAD, ENTRETENIMIENTO INFANTIL 


\section{ABSTRACT}

With emergence and development of technology, Internet has become protagonist of developed societies, in addition, he is presenting a panorama where educational and technological plane merge. In the area of new screens tablets have implemented a strong presence in the day to day of children as an user-friendly tool but, are tablets educational tools? Currently, almost eighty percent of children frequently use this new device for entertainment which gives them autonomy and freedom to access to a multitude of contents; therefore, we concerned that children won't find educational contents.

In this study, it is analyzed the application 'Neox Kidz' as it is considered as a space with audiovisual content created for children. The analysis of content of each element will be able to demonstrate that this app is a safe space that shows cartoons, series and programmes with an educational purpose based on quality entertainment.

KEYWORDS: TECHNOLOGY, EDUCATION, TABLETS, QUALITY, CHILDISH ENTERTAINMENT.

\section{LOS MEDIOS DE COMUNICACIÓN COMO EDUCADORES SOCIALES}

La capacidad de los medios de comunicación de actuar como agentes potenciales de socialización crea expectativas en torno a la construcción de unos contenidos con una finalidad educativa, informativa y de entretenimiento pero no todo lo que se crea y emite es loable; preocupa qué se cuenta y cómo se cuenta.

Los medios de comunicación obtienen la aceptación social porque conectan con dimensiones plenamente personales, profundizan, plasman o analizan la vida de las personas, sus problemas, sus sentimientos y pasiones y lo hacen con tal fuerza que llega al mundo interior del espectador despertando pensamientos, valoraciones, cambios de actitud y percepción de la realidad. Se presentan como si solo pretendieran entretener, distraer y divertir constituyéndose éstas las principales razones por las que el público los consume sin buscar otra pretensión pero que no busque no quiere decir que no encuentre, compruebe o experimente. De un modo sutil se inculcan ideas y se influye en las conductas de los espectadores. Esta visión socializadora los hace ser considerados según Morduchowicz (2004) como "pedagogías públicas". Aguilar 
por su parte afirma que "ese mundo tan radicalmente construido, tiene una gran fuerza persuasiva. Puede influirnos más que lo que nos influyen los hechos reales. Las imágenes gozan de un extraordinario poder emotivo" (AGUILAR, 1998, p.47).

Si nos detenemos ante la audiencia infantil la influencia de los medios les alcanza de manera directa y cada vez más temprana. En la particularización de este tipo de público son inevitables dos puntualizaciones: 1) los niños y niñas tienen una exposición más continuada a los medios de comunicación que los adultos y lo hacen desde el principio de su vida consciente y 2) las niñas y niños se enfrentan a ellos en una época de formación física, desarrollo mental y creación de hábitos y actitudes. No solo asimilan una serie de elementos de los medios sino de todos los ámbitos que lo rodean (familia, escuela, grupo de amigos...) pero no todos ellos son comparables y pueden ser compartidos por sus compañeros en tanto que los medios constituyen un mundo generalizable propiciando identificaciones colectivas. Así, Zires considera fundamental conocer cómo el niño o niña interpreta, elabora e integra los mensajes recibidos a través de los medios de comunicación social; es una tarea indispensable para poder plantearse el papel que éstos juegan en su vinculación con el medio cultural que les rodea y la etapa de desarrollo que están viviendo (zIRES, 1983, p.136).

Más allá de entender a los medios como espacios informativos y de entretenimiento han de contemplarse desde una perspectiva educacional e incluso formativa para el público que los consume. Los medios educan y por ello preocupa especialmente la infancia. Según Yolanda Neira, psicóloga especializada en la etapa infantil, los medios de comunicación son agentes de socialización y como tal el papel que juegan en el desarrollo de los niños y niñas es de elevada importancia. Ante la inquietud por el tipo de contenidos que consume el público infantil Núñez Domínguez (2011) defiende que ante la peligrosidad de los contenidos la formación de la audiencia es una garantía. En ese sentido se consideran potentes medios al cine o la televisión (AGUILAR, 1998; NÚÑEZ DOMÍNGUEZ, 2008) aunque cada vez ha de prestársele mayor atención al medio Internet. A través de los medios se aprenden y aprehenden conductas que influyen de un modo particular en la audiencia infantil como un público permeable y vulnerable. De hecho, no resulta llamativo que las niñas y niños definan e identifiquen a sus más allegados con los personajes que aparecen en la pantalla e imiten determinados comportamientos. De ahí la obsesión por desarrollar contenidos infantiles con una finalidad educativa $\mathrm{y}$ de entretenimiento basados en valores sociales. 


\subsection{EL MEDIO WEB: UN NUEVO ESPACIO PARA LOS CONTENIDOS}

La televisión, desde su nacimiento, ha sido el medio que mayor índice de audiencias ha captado pero la aparición del medio web ha traído consigo la implantación de un nuevo ecosistema mediático revolucionando todo lo establecido hasta ahora en el ámbito de la ecología de los medios, así como el modo en que los individuos nos relacionamos con éstos. "Estamos pasando de los medios de masas a la masa de medios. Pasamos del sistema media-céntrico al yo-céntrico, donde el individuo se transforma en un microorganismo al tener el poder de comunicarse, de intercambiar información, de redistribuir..." (alves, 2010)1 . Como en su momento apuntara Mcluhan (1969): "el contenido de cualquier medio o vehículo es siempre otro medio o vehículo" (p.22) pero Internet supera esta realidad: más que el contenido del medio anterior, Internet incluye los contenidos de todos los medios antecedentes. Es este factor el que justifica su éxito pues si "la hibridación de medios libera gran fuerza o energía como la fusión” (MCLUhAN, 1969, p.67) cuanto más grande sea el número de formas que se funden más alta es la energía producida y, por tanto, mayor es el atractivo para los seres humanos (CANAviLHAs, 2011, p.17).

En las últimas décadas hemos asistido a una extraordinaria expansión de las tecnologías de la información y la comunicación y, en especial, de Internet. En este modelo de sociedad, todo lo relacionado con las TIC desempeña un papel sustancial. Su extraordinaria expansión se traduce en una herramienta imprescindible para el desarrollo individual y colectivo de los pueblos. Así, tal y como afirman Marí Sáez y Sierra (2009): "hoy más que nunca es el ciudadano quien de consumidor a creador cultural, protagoniza las transformaciones del nuevo ecosistema mediático" (p.131). Con el surgimiento de este nuevo medio la tecnología se convierte en la protagonista de las sociedades desarrolladas y nos ubica ante a un panorama en el que se cruza el plano de lo educativo y lo tecnológico. Gutiérrez Martín (2003) expone que Internet como agente educativo que es, al igual que cualquier otro medio de comunicación, sus contenidos y su filosofía nos ayuda a configurar una determinada visión de las distintas realidades. En su relación con la educación, Internet también comparte con otros medios, como la televisión, la radio, los videojuegos o la prensa su doble condición de objeto de estudio y recurso didáctico. Sin embargo, y sin necesidad de esperar al grado de desarrollo que

1. http://elpais.com/diario/2010/09/05/domingo/1283658757_850215.html. Consultado fecha: 05/03/2015

50| ISSN 1696-2079 
permita la inmersión en mundos virtuales, Internet ya ha demostrado, a diferencia de medios anteriores, que puede ofrecer algo más a la educación y a la alfabetización: nuevos entornos de aprendizaje, no solo como apoyo, sino como alternativa (GUTIÉRREZ MARTín, 2003, p.209).

La influencia de esa tecnología empieza a percibirse en nuestras generaciones más jóvenes surgiendo así nuevos perfiles de consumidores. Prensky en su ensayo titulado "The death of command and control?" (2004) acuña dos términos los cuales definen una nueva tipología de consumidores: "inmigrantes digitales" y "nativos digitales". El primero de ellos hace referencia a aquellas personas que se han adaptado a la tecnología. Oscilan entre los 35 y 55 años y son fruto de un proceso de migración digital que supone un acercamiento hacia un entorno altamente tecnificado, creado por las TIC. Por su parte, a los "nativos digitales" los identifica con aquellas personas que han crecido y desarrollado con la era digital. Son usuarios permanentes de las tecnologías con una habilidad consumada. Sienten atracción por todo lo relacionado con lo tecnológico con lo que satisfacen sus necesidades de entretenimiento, diversión, comunicación, información e incluso de formación. Estos nuevos usuarios enfocan su trabajo, el aprendizaje y los juegos de nuevas formas: absorben rápidamente la información multimedia de imágenes y vídeos, igual o mejor que si fueran textos; consumen datos simultáneamente de múltiples fuentes, son multitarea. Afrontan distintos canales de comunicación prefiriendo los formatos gráficos a los textuales y utilizando el acceso hipertextual en vez del lineal. Esperan respuestas instantáneas, permanecen comunicados permanentemente y crean también sus propios contenidos dando lugar a la figura del prosumidor. Estos nuevos consumidores entienden la tecnología como parte integral de sus vidas. Castells (2009) mantiene que como el valor de la red se incrementa exponencialmente en proporción a su número de nodos, la capacidad de comunicación y de información comunicada se ha incrementado en las dos últimas décadas en mayor medida que en toda la historia precedente. Y como en las tecnologías de red los usuarios también son los productores de la tecnología, los jóvenes actuales se han apropiado la comunicación digital, generando nuevas formas de relación, nuevos usos y nuevos hábitos (CASTELLS, 2009, p.112).

Desde el área de la psicología, el nativo digital en su niñez ha construido sus conceptos de espacio, tiempo, causalidad, identidad, memoria y mente a partir, precisamente, de los objetos digitales que le rodean, pertenecientes a un entorno altamente tecnificado. García, Portillo, Romo y Benito (2005) plantean que el crecimiento en este entorno tecnológico puede haber influido en la evolución del cerebro de aquellos individuos. En concreto, se investiga 
el efecto de los juegos electrónicos en algunas habilidades cognitivas y la generación incluso de una nueva estructura neuronal en los individuos. Sin duda, su actividad con la tecnología configura sus nociones sobre lo que es la comunicación, el conocimiento, el estudio/aprendizaje e incluso sus valores personales (GARCía et al., 2005, p.2).

Con la migración de los contenidos televisivos a la red se desdibujan las fronteras y se amplían horizontes en lo que al acceso y uso de los contenidos se refiere en pos de una e-democratización del conocimiento. Cuando solo existía la televisión como medio masivo el espectador se adecuaba a una programación preestablecida, consumía unos contenidos ajustados a una determinada franja horaria; ahora en cambio, los usuarios de Internet acceden a los productos audiovisuales en el momento o situación que más les convenga. "La convergencia implica un cambio tanto en el modo de producción como en el modo de consumo de los medios" (JEnkins, 2008, p.26-27). Prensky expone que los jóvenes del siglo XXI no pueden aprender, entretenerse o divertirse bajo los mismos sistemas que los jóvenes de ayer porque son diferentes sus cerebros y sus culturas (PRENSKY, 2001, p.3). Por ello, hoy son las nuevas tecnologías las que bajo las directrices de lo educativo, el entretenimiento y la diversión deben crear espacios destinados a este público sin olvidar que la fácil accesibilidad a los contenidos requiere que, en el entorno web, se implanten medidas de control incidiendo de un modo acentuado en los productos destinados a los usuarios más jóvenes. Nos encontramos pues, ante un panorama en el que convive la educación con la nueva cultura juvenil, y es por ello por lo que Giroux (1996) expuso que "los educadores tienen que lograr que lo pedagógico sea más político atendiendo tanto a las condiciones, a través de las cuales enseñan, como a lo que significa aprender de una generación que está experimentando la vida en una forma muy diferente de las representaciones que ofrecen las versiones modernistas de escolaridad" (p.15). "Las nuevas tecnologías representan terrenos interactivos que traspasan los límites del lenguaje y la cultura, sin requisitos narrativos, sin complejidades de personaje. La complejidad narrativa cedió su lugar a la complejidad de diseño; el argumento cedió su lugar al ambiente sensorial" (PARKES, 1994, p.50).

Las posibilidades que actualmente brinda el acceso a las redes han modificado los hábitos de las personas. Kofi Annan, Secretario General de la ONU, en la Cumbre Mundial sobre la Sociedad de la Información (2003) afirmó que "las tecnologías de la información y la comunicación no son ninguna panacea ni fórmula mágica, pero pueden mejorar la vida de todos los habitantes del 
planeta"2 . Parece importante llegar a comprender que el problema no radica en la tecnología en sí misma, éticamente neutra, sino en el uso que se haga de ella. En el caso de los colectivos más desprotegidos, los menores principalmente, la responsabilidad sobre las buenas prácticas en el uso de estas herramientas recae en padres, madres y educadores, que deben optar por apoyar el buen empleo de las TIC con sus conocimientos y su buen sentido. Entre las indudables ventajas que ofrecen las tecnologías de la información, desde las instituciones públicas, se puede y debe colaborar en transmitir a todos los responsables de la educación de las y los menores pautas de actuación fiables y seguras que ayuden a formar a nuestros jóvenes en aspectos tan relevantes como la selección de información y contenidos educativos y de calidad así como los peligros del contacto indiscriminado con personas desconocidas o la necesidad de mantener controlado el acceso a nuestra privacidad.

Según Sierra Caballero la investigación en educación comunicativa carece de una evaluación integral sistematizada, así como de un volumen de estudios que arrojen datos comparativos entre países, medios y tipos de públicos en la materia; en todo este contexto falta un compromiso detallado y compartido sobre las competencias que, desde la perspectiva social, son necesarias en la era digital. "Las transformaciones aceleradas del ecosistema informativo exigen de nuestra parte pensar qué entendemos por competencia digital, en qué consistiría el uso crítico de los medios electrónicos para el trabajo, el ocio y el aprendizaje permanente a lo largo de toda la vida" (SIERRA CABALLERo, 2009, p.24).

\subsection{EDUCACIÓN Y CALIDAD EN LAS APPS PARA LA INFANCIA}

Ya en 2007 Castells, Fernández-Ardevol, Qiu y Sey llevaron a cabo un estudio con el objetivo de conocer hasta qué punto la posesión de un móvil conectado se convertía en un bien preciado para los jóvenes, y es que la autonomía comunicativa les permitía construir su propio mundo. Recientemente se ha insertado en nuestras vidas un instrumento que, junto con los smartphones, empieza a hacer sombra a la televisión incluso a los ordenadores portátiles en lo referido al uso y consumo de contenidos audiovisuales en la red: las tablets. Éstas comienzan a configurarse como una herramienta edu-comunicativa de fácil manejo por poseer las siguientes características: a) funcionalidad táctil (un sencillo sistema para los más pequeños), b) un tamaño de pantalla óptimo

2. http://www.itu.int/wsis/messages/annan-es.html. Consultado fecha: 10/03/2015 
para el consumo de contenidos audiovisuales y c) la capacidad de transportarla a cualquier sitio. Plowman y Stephen planteaban en 2005 que la etapa preescolar de los niños es un periodo interesante para investigar la educación a través de las nuevas tecnologías observando la relación entre el aprendizaje formal e informal. Especialistas en marketing ${ }^{3}$ exponen que el $10 \%$ de los niños y niñas menores de un año ya utilizan las pantallas inteligentes, el porcentaje se eleva al $39 \%$ en el caso de los menores de cuatro años y supera el $50 \%$ en los mayores de ocho años. Siete de cada diez menores de doce años conviven y han utilizado ya este instrumento. La mayor parte de los niños y niñas utilizan las tablets con la aprobación de sus tutores. El 22\% de los padres y/o madres reconoce prestar a sus hijos sus smartphones y tablets para mantenerlos ocupados mientras ellos realizan otras tareas. En cuanto al uso que los más pequeños realizan de ella, el $77 \%$ las utiliza para jugar, el $57 \%$ lo hace con propósitos educativos, el $55 \%$ para entretenerse durante los viajes, el $33 \%$ para ver películas y programas de televisión, el $41 \%$ para divertirse en restaurantes o eventos y el $15 \%$ para comunicarse con los amigos y la familia. El $46 \%$ de las aplicaciones descargadas son juegos que promueven únicamente la diversión, el $42 \%$ son juegos educativos, el $28 \%$ promueven la creatividad del pequeño y el $19 \%$ son apps basadas en personajes televisivos. Ante todo ello: ¿son las tablets las nuevas niñeras? ¿Son un medio seguro para el consumo infantil? ¿Encuentra el público infantil contenidos educativos y de calidad en las aplicaciones dirigidas a este target?

En primer lugar, es necesario comenzar definiendo qué se entiende por contenidos educativos y contenidos de calidad. Se considera que el contenido es educativo cuando presenta un discurso en el que se aportan nuevos saberes y se fomentan los valores sociales buscando que el niño o niña adquiera nuevos conocimientos y potencie los que ya posee. En el ámbito multimedia, además de éstos, habría de añadirse otros factores como la usabilidad ${ }^{4}$, la interactividad y la no linealidad pues tal y como indica Gutiérrez Martín (2003): "es necesario tener en cuenta la importancia de un discurso tecnológico el cual trata de magnificar la importancia de la tecnología digital en nuestras vidas y en la educación de nuestro tiempo" (p.198). De este modo, el niño o niña accede al escritorio de la tablet y selecciona la aplicación descargada

3. http://www.marketingdirecto.com/especiales/mobile-marketing-blog/\%C2\%BFson-las-tabletas-lasnuevas-nineras-7-de-cada-10-ninos-usan-ya-estos-dispositivos/Consultado fecha 09/03/2015

4. Según Jakob Nielsen la usabilidad es un atributo de calidad que evalúa la facilidad de uso de una interfaz por parte de un usuario. http://www.nngroup.com/articles/usability-101-introduction-tousability/Consultado fecha 05/03/2015 
teniendo la posibilidad de navegar por su menú desplegable donde encontrará las distintas pestañas con diferentes contenidos otorgándole la libertad de elegir el programa que en ese momento desee, acceder a otros a través de enlaces e incluso consumirlos sin la necesidad de estar sujeto a la lógica de la secuencialidad de la narración.

Contemplando la calidad como un concepto multifactorial, García Avilés (1996) expone que "se habría de considerar criterios operativos sobre la producción y la programación desde una perspectiva que abarque los aspectos estéticos-creativos, prácticos y éticos” p.125). Blanco Mallada (2005) establece que en base a esta definición y teniendo en cuenta los diversos puntos de vista propuestos por profesionales, críticos e investigadores se pueden contemplar las siguientes perspectivas sobre los estándares de calidad: diversidad e interés público, rendimiento económico, ética y profesionalidad (p.309). Por su parte, Tur Viñes (2005) considera que "la calidad de los contenidos audiovisuales dirigidos a la infancia no debe concentrarse solo en el estudio de los efectos de la programación en este público, aunque esto es algo necesario pero no suficiente para valorar la calidad del audiovisual" (p.317318). Además, junto a Ramos Soler sostienen que los contenidos de calidad dirigidos a la infancia deben reunir los siguientes requisitos: "calidad en cuanto a los aspectos audiovisuales, es decir, los recursos técnicos empleados en su creación; respecto al mensaje que transmiten; desde la perspectiva de la legalidad y finalmente desde el punto de vista del entretenimiento" (TUR VIÑES Y RAMOS SOLER, 2008, p.3).

En el ámbito internacional las líneas de investigación y los estándares más relevantes en torno a la medición de calidad de los contenidos dirigidos a la infancia son:

- The cts (Children's Television Standards) de la ABA (Australian Broadcasting Authority) de 1979. La ACTF (Australian Children's Television Foundation) viene estudiando este tema desde 1982. Se centra en los contenidos realizados especialmente para la infancia, el entretenimiento, la calidad técnica así como su deber por ampliar la comprensión y la experiencia de niños y niñas.

- The Annenberg Public Policy Center (APpc) en Pensylvania (eeuu), desde 1995, contempla los contenidos en relación a una edad apropiada, contenidos prosociales y constructivos integrados en las historias narradas, los recursos técnicos, narrativos y creativos así como la pluralidad de personajes.

- La CNTV (Consejo Nacional de Televisión) en Chile, desde 1997, 
plantea la ausencia de violencia, de contenidos sexuales, lenguaje inapropiado, contenidos educativos y conductas constructivas en aras de una integración social.

- El Sistema CEo (sistema de medición de la calidad adoptado por la televisión francesa) contempla los siguientes parámetros: cultura viva, valor de distracción, originalidad del tema, cultura tradicional, calidad de realización, accesibilidad, actualidad, aportaciones de contenidos y originalidad de la forma.

- En Argentina el conacai ${ }^{5}$, (Consejo Asesor de la Comunicación y la Infancia) propone la creación de catorce criterios para la mejora de la programación. Estos son: promoción, protección y defensa de los derechos de los niños, niñas y adolescentes; evitar la morbosidad, las escenas crueles y violentas; respeto y diversidad de las diferentes regiones y diversidad de género; fomento de la creatividad y la dignidad; invitar a la capacidad y reflexión crítica; impulso de la diversión; contenidos que permitan dar voz propia a los niños, niñas y adolescentes; difundir mensajes saludables; respetar a las audiencias; fomentar vínculos con sus comunidades; valores ciudadanos y contemplar los aspectos de la producción del contenido.

Siendo, por tanto, el medio web el más utilizado y con él el consumo de sus contenidos audiovisuales a través de las tablets como herramienta emergente preocupa el contenido audiovisual destinado a los niños y niñas. Tomando como base la díada público infantil y programación educativa en este trabajo se va a analizar la aplicación Neox Kidz, la cual se presenta de la mano de Antena.Neox, como una de las aplicaciones actuales que contienen series, dibujos animados y programas de entretenimiento destinados a la infancia.

\section{OBJETIVOS}

"Los niños prefieren el móvil, los videojuegos e Internet a la televisión" titulaba el diario El País en 20056 . Según Reig (2005): “éstos se decantan por la red frente a la televisión tradicional. En ésta se sienten más libres, más

5. Foro Parlamentario por la Infancia de la República Argentina. "Criterio de calidad de contenidos audiovisuales para niñez y adolescencia”. Consejo Asesor de la Comunicación Audiovisual y la Infancia (CONACAI).

6. Titular periódico El País. Consultado fecha 23/03/2005. 
independientes e interactivos; otra cosa es que lo sean realmente o que esa supuesta libertad entre dentro de lo virtual" (p.65). Habiéndose configurado las tablets como herramientas edu-comunicativas y Neox Kidz como un espacio audiovisual para las niñas y niños en el que éstos, como nativos digitales, se mueven libremente por la aplicación eligiendo de manera autónoma el contenido que desean consumir, el objetivo del presente trabajo es comprobar que dicha app se presenta como un espacio seguro con contenidos audiovisuales educativos y de entretenimiento.

Para poder establecer estándares que permitan medir la finalidad educativa y la calidad de los contenidos de la aplicación Neox Kidz se ha establecido previamente una serie de valores los cuales constituyen la base. Estos son: 1) la educación: refiriéndose tanto a la educación formal, a través de la cual se aporta enriquecimiento personal, cultural y cognitivo; como una educación informal con contenidos que ofrezcan juegos, moralejas, metáforas o utilicen una comunicación directa con los usuarios haciéndolos partícipes de la trama y permitiendo la interactuación. Se busca, por tanto, que el usuario adquiera nuevos conocimientos y potencie los que ya posee, sintiéndose a veces que forman parte del mismo escenario que su personaje favorito, lo cual despierta la atención e interés en el menor; 2) la igualdad de género y la no violencia en cualquiera de sus variantes; 3 ) valores sociales, morales y éticos y 4) el valor del entretenimiento estando presente en productos que son atractivos y persuasivos para los niños y niñas. Espacios de ocio que permiten el descubrimiento de experiencias a veces mediante juegos y generalmente a través de historias potenciando la diversión y por ende, el deseo por ver el programa.

\section{METODOLOGIAS}

Tomando como base el objetivo planteado, el presente trabajo emplea un sistema de investigación de corte cualitativo con el que se pretende alcanzar, a través de un proceso inductivo, un estudio detallado de las unidades fijadas. Este método de trabajo ha permitido desarrollar un análisis dividido en tres fases.

En primer lugar se ha procedido a seleccionar en la televisión aquellos canales dirigidos a un target infantil los cuales tengan presencia en Internet. Tras la puesta en marcha del Código de Autorregulación, y puesto que los medios públicos tienen el deber de velar por la emisión de unos contenidos de calidad, interesa conocer qué sucede actualmente con las entidades pri- 
vadas y su actuación en el medio web. Tras el rastreo llevado a cabo entre los canales de televisión privados en España como Antena 3 y Telecinco se encuentran Boing y Neox Kidz como espacios exclusivos para los niños y niñas. Finalmente se elige el último por pertenecer al grupo AtresMedia el cual muestra un compromiso directo con la educación infantil y la calidad de los contenidos audiovisuales, ejemplo de ello son iniciativas llevadas a cabo como "Objetivo Bienestar Junior" o "El Estirón"; como afirma Cebrián (2004): "es imprescindible la coherencia de cada contenido con la definición global del canal” (p.108).

En segundo lugar, se procede a comprobar si dicho canal está presente en Internet. Se busca que el público infantil cuente con una app concreta que le permita acceder a toda la programación mediante un solo clic. Habiendo comprobado que existe una aplicación dedicada exclusivamente al público infantil: Neox Kidz en su app para tablets, posteriormente se analiza la estructura que ésta presenta. En su menú desplegable el contenido se divide en cuatro pestañas:

Pestaña 1: "Dibujos y Series". En ella se encuentran los dibujos animados y las series de ficción.

Pestaña 2: "Programas". Contiene programas de entretenimiento propios de la cadena de televisión Antena 3.

Pestaña 3: "Learn". Presenta dibujos animados en inglés.

Pestaña 4: "Padres". En esta pestaña se explica a los padres y madres la seguridad, fiabilidad y calidad de dicha aplicación.

Dicha app presenta dos bloques: uno dirigido a bebés y otro a un público infantil de entre 5 y 12 años. Puesto que interesa conocer los contenidos que visiona el usuario infantil cuando él mismo se predispone a consumir programas o dibujos animados, en este trabajo no se ha analizado la opción "Neox Kidz Baby!" pues al ir dirigida a un público aún bebé son los adultos quienes elegirán el programa que más oportuno consideren.

La tercera parte del procedimiento metodológico ha consistido en aplicar, a cada uno de los títulos que contempla la aplicación, una ficha de análisis de contenido que arroje datos sobre el objetivo marcado. Respecto a la elección de la técnica de análisis de contenido Clemente (1992) considera que esta es una buena herramienta de investigación: "el análisis de contenido, por sus características de técnica desarrollada desde los inicios de las investigaciones sobre comunicación, se ha convertido, casi con exclusividad, en la forma de determinar la influencia que los medios de comunicación de masas ejer- 
cen sobre la conducta humana" (p.171). De la misma manera, y siguiendo a Bardin (1996), el análisis de contenido es entendido como "el conjunto de técnicas de análisis de las comunicaciones tendentes a obtener indicadores por procedimientos sistemáticos y objetivos de descripción del contenido de los mensajes permitiendo la inferencia de conocimientos relativos a las condiciones de producción/recepción (contexto social) de estos mensajes" (p.32).

De este modo, y aplicándolo al caso planteado, el análisis de contenido ha permitido valorar:

Cuestiones de carácter general como el diseño y la estructura de la aplicación así como el tipo de programa que se expone: dibujos animados, series de ficción o programas de entretenimiento.

Cuestiones de carácter más específico como el discurso narrativo de los contenidos así como los valores asociados a cada uno ellos.

Para poder verificar que se trata de títulos educativos y de calidad, se tomará como base los estándares propuestos por Australia, Chile, Estados Unidos, Francia y Argentina, propios de una adecuada programática televisiva. A continuación, se expone la creación de cuatro categorías, de elaboración propia, con las que evaluar los contenidos de esta app, las cuales contemplan distintos campos de estudio:

Categoría 1. Aspectos técnicos: calidad a nivel de producción, imagen, sonido, edición y guión (ver tabla nº 1 ).

Categoría 2. Finalidad: educativa (tanto formal como informal), informativa o entretenimiento (ver tabla $\mathrm{n}^{\circ} 2$ ).

Categoría 3. Contenido (ver tabla no 3 ).

- Calidad del discurso (narrativo, lingüístico, creativo, prosocial y constructivo).

- Fomento de la diversidad cultural.

- Igualdad de género.

- Ausencia de violencia.

Siendo estas categorías aplicables del mismo modo al medio televisivo, tal y como lo proponen los países citados, al tratarse este caso de estudio de unos contenidos insertos en el ámbito digital es necesario incluir una cuarta categoría (ver tabla ${ }^{\circ}{ }_{4}$ ) basada en la usabilidad. En ella se contemplarán elementos como la interactividad y la no linealidad de los contenidos. 


\section{RESUlTADOS}

Neox Kidz en su aplicación para tablets contiene un total de treinta y tres programas. En cuanto a sus contenidos en la sección "Dibujos y Series" se han analizado los siguientes títulos: "Angry Birds", "Piggy tales", "Stella", "Rabbids, la invasión", "Súper 4", "Sam y Cat", "WWE Slam City", "Larva”, "Lalaloopsy", "Pet Alien", "Oscar's Oasis", "Iron Kid”, "Campamento Lakebottom", "Pippi Calzaslargas", "El Hombre Invisible", "Papawa", "Capeta", "Supermodels", "Historias de fútbol", "Academia de Gladiadores" y "Yolanda, la hija del Corsario". En la pestaña "Learn" se encuentran los siguientes títulos: "The Mysteries of Alfred Hedgehog", "Pok and Mok", "Toto Trouble” y "Matt's Monster". Por su parte, los títulos correspondientes a la sección "Programas" son los siguientes: "La ciencia de Marrow", "El hormiguero. Los famosos se divierten", "Cámara Kidz. Jandro sorprende a los peques”, "Hazte Eco”, “Tu cara me suena mini. Las mejores actuaciones”, "Me resbala. El teatro pendiente", "Objetivo bienestar Junior" y "Mundo curioso".

Una vez visionados y analizados cada uno de los títulos anteriormente mencionados, y considerando las categorías de medición establecidas, se han obtenido los siguientes datos los cuales dan respuesta al objetivo planteado.

TABLA N ${ }^{\circ}$ 1. CATEGORÍA 1: ASPECTOS TÉCNICOS

\begin{tabular}{|l|l|}
\hline Aspectos técnicos & Total de series/ programas que reúnen estos requisitos \\
\hline Producción & 33 \\
\hline Imagen & 33 \\
\hline Sonido & 33 \\
\hline Edición & 33 \\
\hline Guión & 33
\end{tabular}

TABLA N ${ }^{\circ}$ 2. CATEGORÍA 2: FINALIDAD

\begin{tabular}{|l|l|}
\hline Finalidad & Total de series/ programas que reúnen estos requisitos \\
\hline Educativa & 3 \\
\hline Informativa & 3 \\
\hline Entretenimiento & 33
\end{tabular}


TABLA $\mathbf{N}^{\circ}$ 3. CATEGORÍA: CONTENIDO

\begin{tabular}{|l|l|}
\hline Contenido & Total de series/programas que reúnen estos requisitos \\
\hline Calidad del discurso & 31 \\
\hline Fomento Diversidad Cultural & 0 \\
\hline Fomento Igualdad de Género & 7 \\
\hline Ausencia de violencia & 31
\end{tabular}

TABLA $\mathbf{N}^{\circ}$ 4. CATEGORÍA: USABILIDAD

\begin{tabular}{|l|l|}
\hline Usabilidad & Total de series/ programas que reúnen estos requisitos \\
\hline Interactividad & 33 \\
\hline No linealidad & 33 \\
\hline
\end{tabular}

\section{CONCLUSIONES}

Tras los datos obtenidos se puede afirmar que el nuevo portal infantil de Atresmedia se ha diseñado basándose en cuatro conceptos: educación, entretenimiento, seguridad y usabilidad ofreciendo todo el contenido de Neox Kidz y una selección de los mejores programas de la cadena. El lenguaje audiovisual ha de considerarse una herramienta básica para la educación y la integración social, por ello, es interesante destacar que la finalidad de esta app es, por tanto, ofrecer un entorno seguro a las madres y padres y un lugar de disfrute para sus hijos e hijas estando en contacto con contenidos actualizados. Neox Kidz en su aplicación para tablets presenta un diseño que ha sido pensado para que sea un espacio atractivo, intuitivo y de fácil manejo de modo que los más pequeños pueden visualizar todo el contenido con solo desplazar el dedo por la pantalla.

Con el análisis del material expuesto en la aplicación, y ajustando los resultados a las categorías previamente fijadas, se ha obtenido que el cien por cien de los títulos cumple con la calidad referida a los aspectos técnicos (categoría 1) y con la usabilidad de dicho espacio web (categoría 4). Todos los contenidos presentan la característica de "la no linealidad" así como el elemento "interactividad" aunque resulta interesante destacar en relación a esta última un punto débil referido a la limitación para experimentar actividades interactivas. En este sentido, la interactividad podría verse completada con la posibilidad de establecer un diálogo entre los usuarios a través de un 
chat donde poder intercambiar opiniones acerca de los distintos títulos. Se les podría ofrecer un espacio dedicado a "concursos" con el que potenciar la fidelidad dándoles la posibilidad incluso de que se conviertan en prosumidores pudiendo subir a la plataforma materiales realizados por ellos mismos. De este modo se experimentaría la globalización haciéndoles partícipes de la ausencia de fronteras favoreciendo, al mismo tiempo, experiencias en las nuevas formas de comunicación virtual con otros usuarios.

Según los datos referidos a la segunda categoría se afirma que educación e información son dos variables que pueden darse a la vez en un mismo programa a través del entretenimiento. El cien por cien de los contenidos es considerado de entretenimiento, solo tres títulos potencian una educación tanto formal como informal a través de programas como "La ciencia de Marron", "Objetivo bienestar" y "Hazte eco"; correspondiendo el mismo número a contenidos exclusivamente informativos: "Objetivo bienestar", "Hazte eco" y "Mundo curioso" cuyos mensajes están relacionados con el cuidado personal, las buenas prácticas sociales, el medio ambiente, el reciclaje o el mundo animal.

En relación a la tercera categoría, el $96,9 \%$ de los títulos presenta calidad del discurso mediante un buen empleo del lenguaje, diálogos cuidados y ausencia de violencia. El resto de los títulos no se clasifican dentro de esta categoría ya que los diálogos utilizados manifiestan la exaltación de la violencia. "WWE Slam City" y "Iron Kid", donde los personajes recurren a los golpes, las patadas y las peleas para alcanzar sus objetivos dan muestra de ello.

En cuanto la diversidad cultural se detecta que no existen títulos que fomenten la representación de otras culturas. En este escenario, y como ejemplo de buenas prácticas para con esta variable, se recomienda la serie de animación de producción catalana titulada "Asha", la cual surgió con la intencionalidad de enseñar a los más pequeños la gran diversidad multicultural.

En lo referido a la igualdad de género es necesario destacar varios aspectos. De un total de 33 títulos, siete series de dibujos animados fomentan la igualdad. Son aquellas en cuyas tramas principales aparecen tanto personajes masculinos como femeninos: "Iron Kid", "Campamento Lakebotton", "Academia de Gladiadores", "The Mysteries of Alfred Hedgehog", "Toto Trouble", "Matt's Monster" siendo "Yolanda, la hija del corsario negro" la única serie cuya protagonista es una mujer la cual muestra características y desempeña acciones "no propias de su sexo": es aventurera y es la capitana de un barco. Solo seis títulos son protagonizados por personajes femeninos: "Sam y Cat", "Lalaloopsy", "Pippi Calzaslargas", "Yolanda, la hija del corsario negro", "Stella" y "Supermodels", potenciándose en esta última los este- 
reotipos de chicas perfectas. En relación a las estereotipias se ha de señalar que los dibujos animados basados en la práctica deportiva como "Capeta" e "Historias de fútbol" asocian el deporte de carreras y el fútbol con el sexo masculino. Además, el programa "Cámara Kidz" contiene un vídeo titulado "Superhéroes" en el que se pretende que los niños conozcan a sus personajes favoritos. Tras su análisis de detecta que solo participan niños y a éstos únicamente se les presentan superhéroes masculinos; se aprecia pues una fuerte ausencia femenina: ¿no existen superheroínas? ¿Es que las niñas no pueden tener entre sus personajes favoritos a un superhéroe? Es importante y necesario llevar a cabo ejercicios de buenas prácticas en aras del fomento de la igualdad.

Finalmente, y habiendo analizado todos los títulos en base a la ausencia de violencia, treinta y uno de ellos se presentan como contenidos no violentos. Solo "WWE Slam City" y "Iron Kid", ya mencionados anteriormente, se clasifican como contenidos violentos puesto que basan sus tramas en acciones donde se recurre a la violencia para alcanzar sus objetivos.

Más allá de estos apuntes, es importante señalar el compromiso de la aplicación Neox Kidz con los inmigrantes digitales: padres y madres que continuamente realizan el esfuerzo por acercarse a las nuevas tecnologías para así poder enseñar y controlar lo que sus hijos consumen, así, esta aplicación ofrece contenidos atractivos que educan y entretienen. En relación a la seguridad, Neox Kidz cuenta con la existencia de un control parental. Este es un sistema de control que permite restringir el tipo de contenido al que pueden acceder las niñas y niños en función de su edad. De esta forma, los más pequeños (de o a 4 años) pueden disfrutar de un entorno seguro con contenidos adaptados a su edad en "Neox Kidz Baby!". Para poder acceder a los contenidos para niños de 5 a 12 años deberán resolver una sencilla fórmula matemática, esto hace que no sea posible cambiar de entorno sin la ayuda de sus tutores.

Entre los estudios precedentes al que aquí se redacta se pueden citar: "La calidad como consecuencia de una buena segmentación de edades en la televisión temática infantil: Playhouse Disney y los contenidos preescolares", "Las nuevas tecnologías y los menores: análisis de las páginas web de los canales temáticos infantiles de televisión digital", ambos trabajos de Melgarejo Moreno y Rodríguez Rosell (2010) y "El compromiso de la televisión pública española por una e-programación infantil de calidad. El portal Clan como una web edu-comunicativa”, de Sánchez-Labella Martín y Guarinos (2011).

Si Sánchez-Labella Martín y Guarinos demuestran el compromiso de la televisión pública en España por la educación a través de los dibujos anima- 
dos en su parrilla televisiva, Melgarejo Moreno y Rodríguez Rosell afirman que en su mayoría los títulos de animación pertenecientes a canales privados como Playhouse Disney, Disney Channel, Kidsco, Disney Cinemagic, Nick, Boomeran, Cartoon Network, Jetix y Baby Firtr, presentes tanto en el medio televisivo como el medio web, mantienen igualmente un compromiso educativo con el público al que se dirige. Estas últimas autoras sostienen la idea de que el desarrollo de las nuevas Tecnologías de la Información y la Comunicación junto a la implantación de Internet han propiciado nuevas formas de comunicación e interacción con la infancia quedando la televisión desplazada por otras formas de ocio a través de la Red: "las nuevas tecnologías han permitido aunar bajo un mismo entorno educación, entretenimiento e información permitiendo al menor acceder a un tipo de información más global y completa, donde se unen la fuerza de la comunicación visual, la sonora y el texto escrito bajo una misma finalidad: mantener al usuario entretenido e informado" (MELGAREJO MORENO Y RODRÍGUEZ ROSELL, 2010, p.12). Pero en referencia a las nuevas pantallas el presente estudio da un paso más allá del consumo de animación a través del ordenador presentando las tablets como un nuevo espacio audiovisual.

De este modo, y subrayando las palabras de las anteriores autoras, se concluye exponiendo que Neox Kidz, con su presencia en las tablets como un nuevo dispositivo de aprendizaje para los niños y niñas, se presenta como un espacio seguro con contenidos audiovisuales de calidad, educativos y de entretenimiento. Se trata, por tanto, de una aplicación e-learning la cual, a través del audiovisual, proporciona una educación tanto formal como informal fomentando de este modo una nueva forma de aprendizaje del público infantil.

\section{BIBLIOGRAFÍA}

AGUILAR, P., (1998), Mujer, amor y sexo en el cine español de los 9o, Madrid, España: Fundamentos.

BARDIN, L., (1996), Análisis de contenido, Madrid, España: Akal.

Blanco Mallada, L. (2005). Estándares de calidad en televisión y su valoración, Revista Comunicar, (25), 309.

CANAVILHAS, J. (2011). El nuevo ecosistema mediático, Revista Index Comunicación, (1), 13-24.

CASTELLS, M., (ED.), (2007), Comunicación móvil y Sociedad, Barcelona, España: Ariel. 
CASTELLS, M. (2009). La apropiación de las tecnologías. La cultura juvenil en la era digital, Revista Telos, (81), 111-113.

CEBRIÁN, M., (2004), Modelos de televisión: generalista, temática y convergente con Internet, Barcelona, España: Paidós.

CLEMENTE, M., (1992), Psicología social: métodos y técnicas de investigación, Madrid, España: Henderán.

GARCÍA AVILÉS, J. A., (1996), "Algunos parámetros del periodismo de calidad en televisión”. En Latorre Izquierdo J.; Vara Miguel, A. y Díaz Méndez, M. (Coords), Ecología de la televisión: tecnologías, contenidos y desafíos empresariales (125133), Pamplona, España: Eunate.

GARCÍA, F., PORTILLO, J., ROMO, J. Y BENITO, M., (2005), Nativos digitales y modelos de aprendizaje, País Vasco, España: Universidad del País Vasco y el Gobierno Vasco.

GIROUX, H. (1996). Educación posmoderna y generación juvenil, Revista Nueva Sociedad, (146), 148-167.

GUTIÉRREZ MARTÍN, A., (2003), Alfabetización Digital. Algo más que botones y teclas, Barcelona, España: Gedisa.

JENKINS, H., (2008), Convergence Culture. La cultura de la convergencia de los medios de comunicación, Barcelona, España: Paidós.

MARÍ SÁEZ, V. M. Y SIERRA CABALLERO, F. (2008). Capital informacional $y$ apropiación social de las nuevas tecnologías. Las redes críticas de empoderamiento local en la Sociedad Europea de la Información, Revista Telos, (74), 126133.

MCLUHAN, M., (1969), Os meios de comunicação como extensões do Homem, Sao Paulo, Brasil: Cultrix.

MELGAREJO MORENO, I. Y RODRÍGUEZ ROSELL, M. M. (2010). Las nuevas tecnologías y los menores: análisis de las páginas web de los canales temáticos infantiles de televisión digital, Revista Área abierta, (27), 1-14.

MELGAREJO MORENO, I. Y RODRÍGUEZ ROSELL, M. M. (2010). La calidad como consecuencia de una buena segmentación de edades en la televisión temática infantil: Playhouse Disney y los contenidos preescolares. Ponencia expuesta en Comunicación y Desarrollo en la Era Digital. Congreso Internacional AE-IC. Málaga 2010.

MORDUCHOWICZ, R., MARCON, A. Y MINZI, V., (2004), El cine de animación, Argentina: Ministerio de Educación, Ciencia y Tecnología.

NÚÑEZ DOMÍNGUEZ, T., (2008), "La mujer dibujada. El sexismo en películas $y$ series de animación”. En Loscertales, F. y Núñez Domínguez, T. (Coords.), La mirada de las mujeres en la sociedad de la información (241-270). Madrid, España: Siranda Visionnet. 
NÚÑEZ DOMÍNGUEZ, T., (2011), "Ideas-clave para la reflexión". En Núñez Domínguez, T. y Troyano Rodríguez, Y. (Coords.), La violencia machista en el cine. Materiales para una intervención psico-social (1-34). Madrid, España: Delta.

PARKES, W. (1994). Random Access, Remote Control: the Evolution of Story Telling, Revista Omni, (1), 50.

PRENSKY, M. (2001). Digital Natives, Digital Immigrants, Revista On the Horizon, 9(5), 1-6.

PRENSKY, M., (2004), The death of command and control?, Montreal, Canadá: Technology Alliance Partners.

PLOWMAN, L. Y STEPHEN C. (2005). Children, play and computers in pre-school education, Revista British Journal of Educational Technology, (2), 145-157.

REIG, R. (2005). Televisión de calidad y autorregulación de los mensajes para niños y jóvenes, Revista Comunicar ${ }_{s}(25), 63-70$.

SÁNCHEZ-LABELLA MARTÍN, I. Y GUARINOS, V. (2011). El compromiso de la televisión pública española por una e-programación infantil de calidad. El portal "Clan" como una web edu-comunicativa. Actas II Congreso Internacional Sociedad Digital: espacios para la interactividad y la inmersión, (2), 599-610.

SIERRA CABALlERO, F. (2009). Capitalismo Cognitivo y Educomunicación. Críticas y retos de las políticas democráticas de socialización del conocimiento. VIII Encuentro Latinoamericano de Facultades de Comunicación Social, 21. La Habana, Cuba.

TUR VIÑES, V. (2005). Aproximación a la medida empírica de la calidad del audiovisual dirigido a niños, Revista Comunicar, (25), 317-318.

TUR VIÑES, V. Y RAMOS SOLER, I. (2008). Diseño de cuestionario y escalas para la medición del entretenimiento percibido, preferencias programa/cadena $y$ hábitos de visionado televiso en niños de 4 a 12 años. Actas y memoria final: Congreso Internacional Fundacional AE-IC, Santiago de Compostela. Revista I+C Investigar a Comunicación, Asociación Española de Investigación de la Comunicación, Santiago de Compostela.

ZIRES, M. (1983). El discurso de la televisión y los juegos infantiles, Revista Comunicación y Cultura, (10), 109-136. 
LAS TABLETS COMO HERRAMIENTAS EDUCATIVAS PARA LA INFANCIA NEOX KIDZ, UN ESTUDIO DE CASO 